\title{
Statistical analysis of ocular monochromatic aberrations in Chinese population for adaptive optics ophthalmoscope design
}

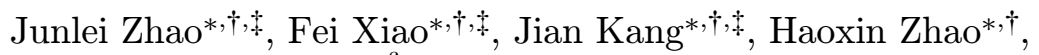 \\ Yun Dai ${ }^{*, \dagger, \S, \|}$ and Yudong Zhang, ${ }^{*, \dagger, \boldsymbol{\top}, \|}$ \\ *The Laboratory on Adaptive Optics \\ Institute of Optics and Electronics \\ Chinese Academy of Sciences \\ Chengdu 610209, P. R. China \\ ${ }^{\dagger}$ The Key Laboratory on Adaptive Optics \\ Chinese Academy of Sciences \\ Chengdu 610209, P. R. China \\ University of Chinese Academy of Sciences \\ Beijing 100049, P. R. China \\ $\S_{\text {daiyunqq@163.com }}$ \\ 『ydzhang@ioe.ac.cn
}

Received 23 December 2015

Accepted 30 March 2016

Published 26 May 2016

\begin{abstract}
It is necessary to know the distribution of the Chinese eye's aberrations in clinical environment to guide high-resolution retinal imaging system design for large Chinese population application. We collected the monochromatic wave aberration of 332 healthy eyes and 344 diseased eyes in Chinese population across a $6.0-\mathrm{mm}$ pupil. The aberration statistics of Chinese eyes including healthy eyes and diseased eyes were analyzed, and some differences of aberrations between the Chinese and European race were concluded. On this basis, the requirement for adaptive optics (AO) correction of the Chinese eye's monochromatic aberrations was analyzed. The result showed that a stroke of $20 \mu \mathrm{m}$ and ability to correct aberrations up to the 8th Zernike order were needed for reflective wavefront correctors to achieve near diffraction-limited imaging in both groups for a reference wavelength of $550 \mathrm{~nm}$ and a pupil diameter of $6.0 \mathrm{~mm}$. To verify the analysis mentioned above, an AO flood-illumination system was established, and high-resolution retinal imaging in vivo was achieved for Chinese eye including both healthy and diseased eyes.
\end{abstract}

Keywords: Ocular aberrations; adaptive optics; retinal imaging.

${ }$ Corresponding authors.

This is an Open Access article published by World Scientific Publishing Company. It is distributed under the terms of the Creative Commons Attribution 4.0 (CC-BY) License. Further distribution of this work is permitted, provided the original work is properly cited. 


\section{Introduction}

It is well known that the human eye suffers from lowand high-order aberrations (HOAs) that greatly degrade retinal image quality. In 1997, Liang et al. built the world's first adaptive optics (AO) system for the eye and obtained photoreceptor-resolved high-resolution retinal images in vivo through HOAs correction. ${ }^{1}$ Since that time a great number of papers and reports in this area were published; a comprehensive and informative review on $\mathrm{AO}$ for high-resolution imaging in the living retina was made by Williams ${ }^{2}$ in 2011. During recent years, many AO high-resolution retinal imaging systems have been established in lab for experimental study, including AO fundus cameras, AO Scanning Laser Ophthalmoscope (SLO) systems and AO optical coherence tomography (OCT) systems. ${ }^{1,3-10}$ Many technological advances have been achieved and a lot of scientific and pathological studies have been done. ${ }^{11-15}$

It is significant to transit the AO retinal imaging system from laboratory to clinical application. Since ocular aberrations have a large individual variability, large amplitudes low-order aberrations (LOA) and small amplitudes HOAs exist simultaneously, ${ }^{16}$ both of which have serious influence on retinal image quality, design of AO retinal imaging system for clinical application is a challenging task. Based on the aberration characteristics of about 100 European healthy subjects, Miller and Roorda reached a conclusion that, for the $7.5-\mathrm{mm}$ pupil, correction of aberrations up to the 10th Zernike order was necessary to reach diffraction-limited imaging in approximately $95 \%$ of the population and the largest peak-to-valley (PV) error representing the most demanding condition for $\mathrm{AO}$ correction was $11 \mu \mathrm{m} .{ }^{17} \mathrm{~A}$ single corrector was hard to simultaneously provide high spatial fidelity and large dynamic range to fully compensate lower and higher order ocular aberrations. To address that issue, a dual deformable mirror (DM) scheme was proposed, one DM with high spatial fidelity and the other with large stroke. ${ }^{18-22}$ With the development of the DM, a voice-coil actuated DM with high spatial fidelity and large dynamic range was developed by Imagine Eyes and Alpao, which had excellent high-order aberrations correction ability with very large stroke. ${ }^{23,24}$ Based on this DM, there are already some commercial AO high-resolution ophthalmoscopes developed by some companies.
It is necessary to characterize the ocular aberrations in clinical environment for the design of $\mathrm{AO}$ ophthalmoscope. Detailed measurements of the spatial distribution of wave aberrations have been made in large populations of healthy, healthy adult eyes with large pupils, ${ }^{25-27}$ including the data collected at 10 laboratories (2560 eyes). ${ }^{28}$ Some research studied the aberration characteristics of diseased eyes ${ }^{29-31}$ including a population of about 24,000 patients from Carl Zeiss Vision's wavefront database. ${ }^{32}$ The subjects of the research mentioned above were mostly European. However, studies showed that the aberrations were dependent on the subjects' racial background. ${ }^{33-35}$ Ocular aberration characteristics of Europeans cannot be directly used to guide AO high-resolution retinal imaging system design for large Chinese population application. A statistical analysis of Chinese ocular aberrations is essential to solve this problem.

In the current work, we first characterized the Chinese eye's monochromatic aberrations including healthy and diseased eyes, and concluded some differences of aberrations between the Chinese and European race. Then the requirement for AO correction of the Chinese eye's monochromatic aberrations was analyzed. To verify the analysis mentioned above, a dual DM AO flood-illumination retinal imaging system was established, and highresolution retinal imaging in vivo was achieved for Chinese eye including both healthy and diseased eyes.

\section{Population Statistics of the Chinese Eye's Monochromatic Aberrations}

The data for ocular aberrations were collected clinically from the patients in West China Hospital (Chengdu, China) and the Eye Hospital of Wenzhou Medical University. The Ethics Committee of West China Hospital and the Eye Hospital of Wenzhou Medical University approved this study and signed informed consent was obtained from all subjects after they were informed of the procedures and possible consequence of this study.

\subsection{Subjects and instruments}

A total of 344 subjects with diseased eyes (age range 22 to 69 years; mean age 41 years; 120 males and 224 females; 108 left eyes and 236 right eyes) were 
recruited as the abnormal group for this study. The eyes in the abnormal group all suffered from ocular diseases such as diabetes retinopathy $(n=110)$, glaucoma $(n=72)$, maculopathy $(n=45)$, retinopathy $(n=51)$, amblyopia $(n=34)$ and diseases which were not accurately diagnosed $(n=32)$. Another group of 332 subjects (age: $43 \pm 14$ years) was recruited as the normal group and admission criterion was that the subject had no ocular pathologic features other than refractive error. A commercial aberrometer OPD-Scan $\Pi$ (NIDEK, Tokyo, Japan) was used to measure monochromatic aberrations in this study.

\subsection{Data collection}

Before measurements, dilation of pupil and paralysis of accommodation were achieved in both healthy and diseased eyes with $1 \%$ cyclopentolate solution. The aberrations of each eye were measured three times and the average result was used. For convenience of subsequent analysis, the ocular aberrations were expressed as their equivalents for a reference wavelength of $550 \mathrm{~nm}$ and a pupil diameter of $6.0 \mathrm{~mm}$. The aberrations were calculated to the 10th Zernike order for each subject using the standards recommended by the Optical Society of America (OSA). ${ }^{36}$

\subsection{Data analysis}

Figure 1 shows the distribution of each Zernike mode in the subjects. The mean values are shown by the symbols. Error bars indicate \pm 1 standard deviation from the mean. As the values of Zernike polynomials higher than 8th order are approximately zero, only the values of the first eight Zernike order are shown in the figure.

Several aspects of the data were noteworthy in both normal and abnormal groups. Firstly, the LOAs (defocus and astigmatism) took up the major part of the aberrations in both normal and abnormal groups. The absolute value of LOAs accounted for over $95 \%$ of the absolute value of total wavefront aberrations in normal group, and the percentage in abnormal group was $80 \%$. This was mainly because more than $90 \%$ of the eyes in normal group suffered from myopia. Secondly, for all high-order Zernike modes except Zernike $Z_{3}^{-1}, Z_{3}^{1}$ and $Z_{4}^{0}$, the mean value was approximately zero and the variability
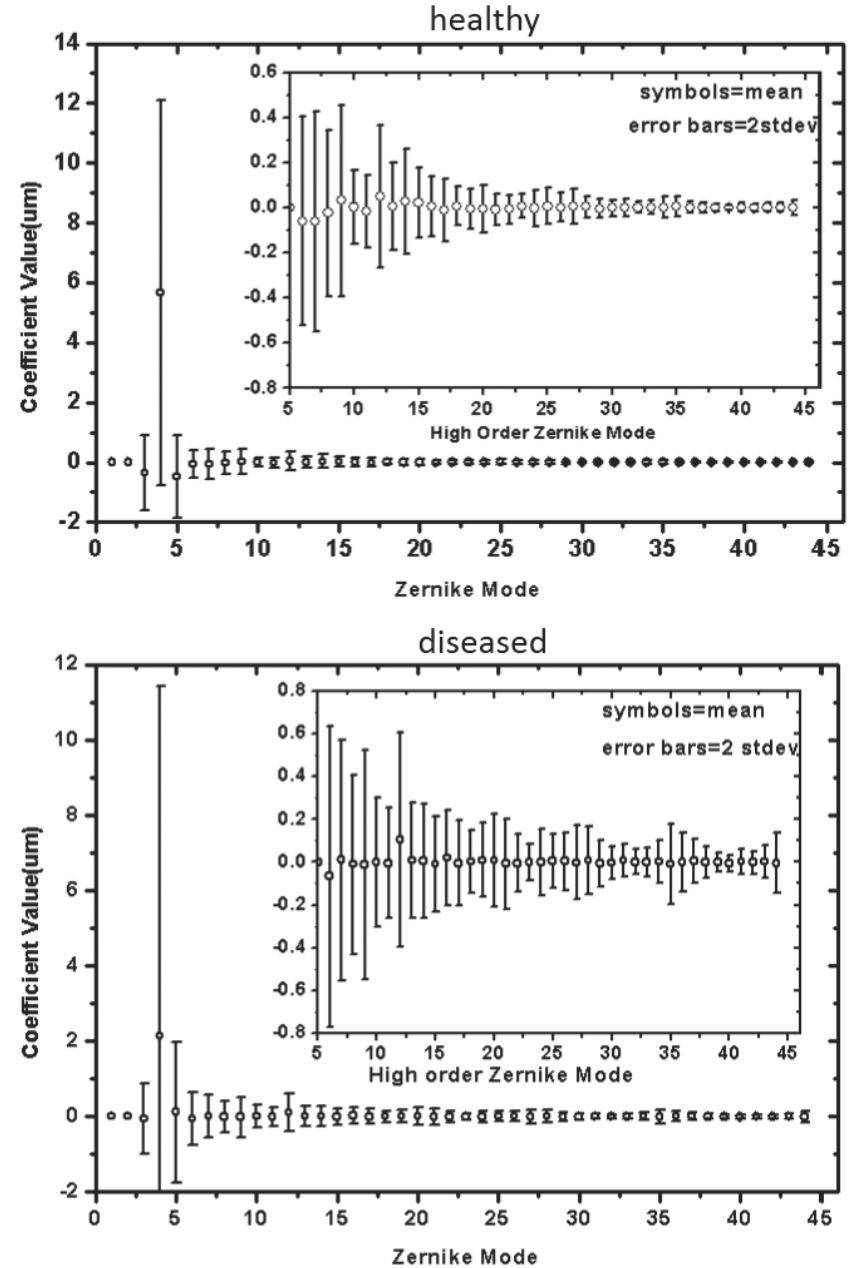

Fig. 1. Mean values of Zernike coefficients from the 2nd to the 8 th order in the normal group and abnormal group across a $6.0-\mathrm{mm}$ pupil. The error bars represent plus and minus one standard deviation from the mean value.

was large. For example, Zernike $Z_{4}^{0}$ in normal group had a mean \pm standard deviation of $0.05 \pm 0.318 \mu \mathrm{m}$, the value in abnormal group was $0.105 \pm 0.503 \mu \mathrm{m}$. In addition, the other HOAs (aberrations higher than 2nd radial order) in abnormal group were significantly larger than those typically found in normal group.

The average values of absolute HOA coefficients across the subjects in both groups are shown in Fig. 2. The sum of absolute coefficients of HOAs in abnormal group was $2.417 \mu \mathrm{m}$ over a $6.0 \mathrm{~mm}$ pupil, which was 1.5 times larger than the $1.594 \mu \mathrm{m}$ in normal group. For each Zernike mode of the HOAs, the ratio of abnormal group to normal group was generally in the range of 1.1 to 4.5 . In addition, the root mean square (RMS) of HOAs was about 


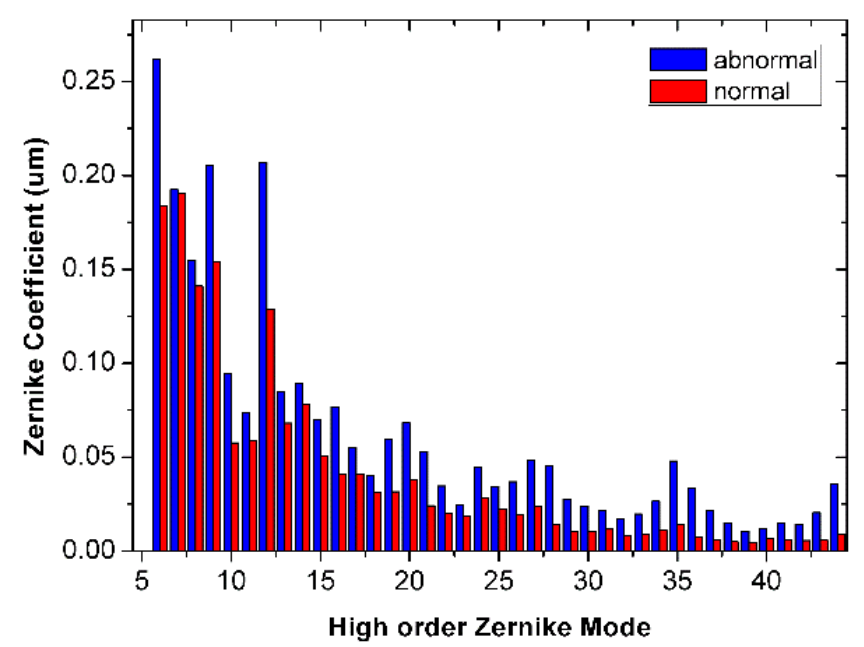

Fig. 2. Mean absolute values of Zernike coefficients from the 3rd to the 8th order in normal group and abnormal group across a 6.0-mm pupil.

$0.7 \mu \mathrm{m}$ in abnormal group, 1.4 times of the value 0.5 $\mu \mathrm{m}$ in normal group.

A great number of papers and reports about the aberrations of European eyes had been published. In this part, we compared the aberrations in the normal group with the aberrations of European eyes that was reported by Porter et $a .^{25}$ The aberrations of European eyes were collected for the $5.7-\mathrm{mm}$ pupil diameter which was very close to the value of $6.0-\mathrm{mm}$ for Chinese eyes. Then we concluded some differences of aberrations between the Chinese and European race. Firstly, the absolute value of LOAs accounted for over $95 \%$ of the absolute value of total wavefront aberrations in Chinese eyes (normal group), which was larger than the value of $92 \%$ in European eyes. This was mainly because more than $90 \%$ of the eyes in Chinese suffered from myopia. Secondly, spherical aberration had a mean value ( \pm one standard deviation) of $+0.05 \pm 0.318 \mu \mathrm{m}$ in the Chinese eyes. However, in European eyes, the value of spherical aberration was $+0.138 \pm 0.103 \mu \mathrm{m}$, and the mean value was about 2.7 times larger than the value in Chinese eyes. Thirdly, Zernike $Z_{3}^{-1}$ and $Z_{3}^{1}$ in Chinese eyes had mean value deviation of $-0.063 \mu \mathrm{m}$ and $-0.024 \mu \mathrm{m}$, respectively, which were all approximately zero in European eyes. In addition, the RMS of HOAs was about $0.31 \mu \mathrm{m}$ in European eyes, which was calculated based on the data reported in the paper. In the Chinese eyes, the RMS of HOAs was about $0.5 \mu \mathrm{m}$, which was almost 1.7 times larger than the value in European eyes. In conclusion, analysis of requirement for $\mathrm{AO}$ correction of the Chinese eye's monochromatic aberrations was needed, because of the difference between the population statistics of Chinese and European eyes.

\section{Requirement for AO Correction of the Chinese Eye's Monochromatic Aberrations}

Two important parameters of the ocular aberrations for AO correction are spatial-frequency and magnitude, which determine the requirements for the stroke and number of actuators of wavefront correctors. A larger stroke is needed to achieve effective correction of large-magnitude aberrations. More actuators with a localized influence function are necessary for the correction of high-spatial-frequency aberrations (i.e., higher order aberrations). ${ }^{17}$

For an AO system, the effective correction of aberrations requires the stroke of the corrector to be no less than the PV value of the aberrations. Figure 3 shows the PV values of total-order aberrations, total-order aberrations without defocus, and HOAs over a $6-\mathrm{mm}$ pupil in both normal and abnormal groups. In normal group, the PV value of the total-order aberrations was $24.1 \pm 10.9 \mu \mathrm{m}$ (mean \pm stdev); the $\mathrm{PV}$ value of the total-order aberrations without defocus was $7.1 \pm 2.5 \mu \mathrm{m}$; the PV value of the HOAs was $3.9 \pm 1.2 \mu \mathrm{m}$. As to abnormal group, the corresponding values were $19.0 \pm 10.05 \mu \mathrm{m}, 9.0 \pm 2.75 \mu \mathrm{m}, 6.3 \pm 1.6 \mu \mathrm{m}$. A stroke

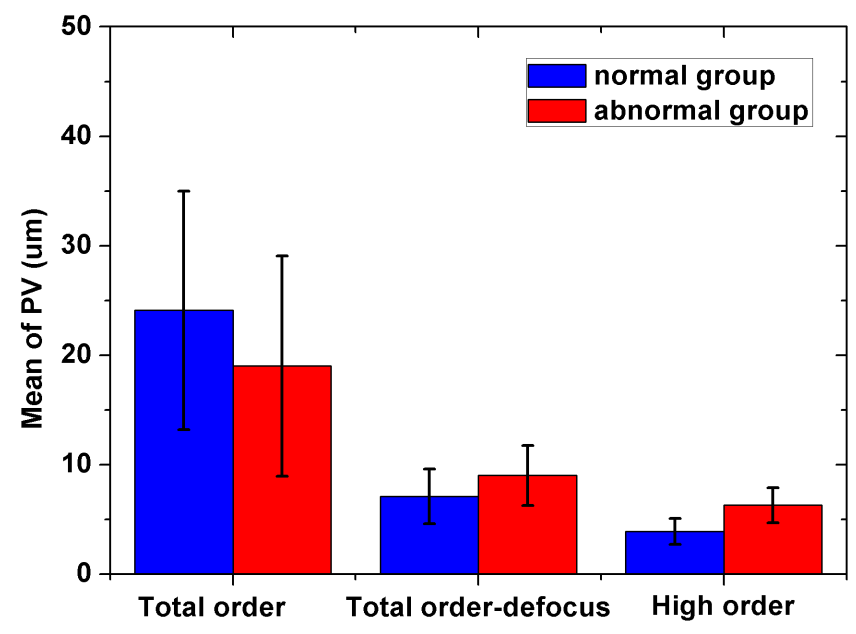

Fig. 3. PV values under three different refraction in both normal and abnormal group. Mean values were shown by the symbols. The error bars in this figure indicated \pm standard deviations from the mean. 


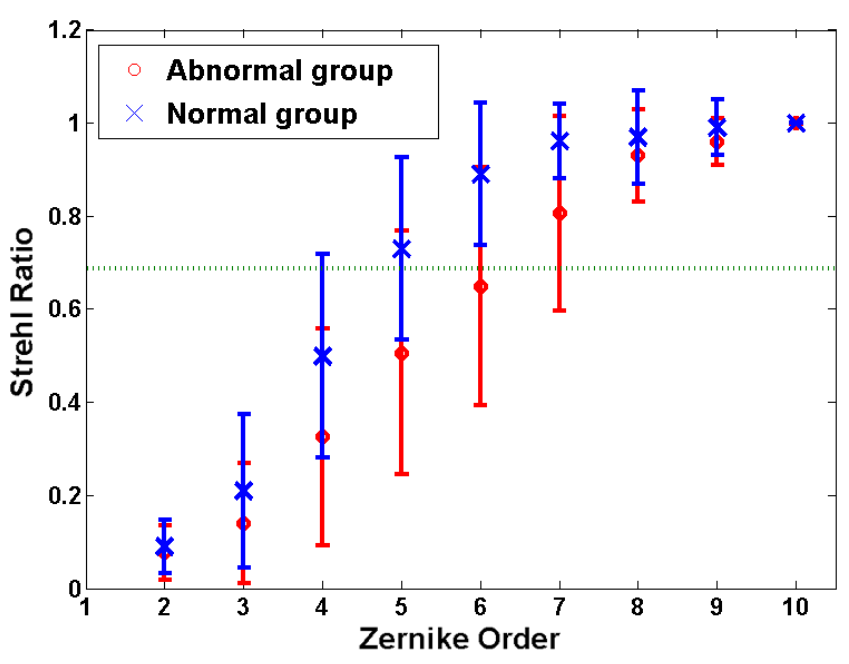

Fig. 4. SR as a function of correction for Zernike order in normal and abnormal group. Mean values were shown by the symbols. The error bars in this figure indicated \pm 2 standard deviations from the mean. Horizontal dashed line corresponds to SR for the diffraction limit.

with maximum deformation of at least $20 \mu \mathrm{m}$ focus (PV) was needed for reflective wavefront correctors such as DM to achieve effective correction of the total-order aberrations for approximately $95 \%$ of the population of both groups.

The distribution of HOAs determines the requirement for actuator number of the wavefront correctors. The Strehl ratios (SR) measured with aberrations corrected up to different Zernike orders in normal and abnormal group are shown in Fig. 4 over a $6.0-\mathrm{mm}$ pupil. Correction of aberrations up to the 7th Zernike order was necessary to reach near diffraction-limited imaging $(\mathrm{SR} \approx 0.8)$ for the normal group, and correction of aberrations up to the 8th order was needed for abnormal group.

\section{AO High-Resolution Retinal Imaging for Chinese Eye}

To experimentally verify that AO system with a stroke of $20 \mu \mathrm{m}$ focus and ability to correct aberrations up to the 8th order could meet the requirement for $\mathrm{AO}$ correction of the Chinese eye's monochromatic aberrations, a dual DM AO floodillumination retinal imaging system was established, and high-resolution retinal imaging was achieved for Chinese eyes in both normal and abnormal groups.

\subsection{AO flood-illumination system}

A single DM in our lab was hard to simultaneously provide high spatial fidelity for correction of aberrations up to the 8th order and large dynamic range with maximum deformation of at least $20 \mu \mathrm{m}$ focus (PV).

To address this issue, the dual DM scheme was adopted, with a 35-element bimorph DM to correct large amplitude, LOAs and a 169-element PZT DM to correct high spatial frequency, HOAs. The 35element bimorph DM had a $20 \mathrm{~mm}$ aperture in diameter and the maximum deformation for focus was $20 \mu \mathrm{m}$, which was described in detail elsewhere, ${ }^{34,35}$ here we would just introduce the 169-element PZT DM. The 169-element PZT DM was based on 3-mm spacing piezoelectric actuators, and it was manufactured successfully in our lab recently. The 3-mm spacing piezoelectric actuators was based on the characteristic of $\mathrm{d} 31$ in PZT. The specifications of a single actuator are as follows: (1) coupling: $14 \%$, (2) maximum deformation: $\pm 2 \mu \mathrm{m},(3)$ maximum voltage: $\pm 500 \mathrm{~V}$. Figure 5 shows the correction capabilities of the 169-element PZT DM. As shown in the figure, the first 45 modes could be corrected effectively, which could fulfil the task of correcting for the first 8th order of ocular aberrations. However, the use of two DMs increased complexity of the optical setup and the overall cost of the system.

With the two DMs above, a dual DM AO floodillumination system was established. The schematic layout of the system is shown in Fig. 6. It consisted of three sub-systems: (1) AO system for correction of the aberrations of the eye and the system, (2) fixation channel for pupil alignment and imaging

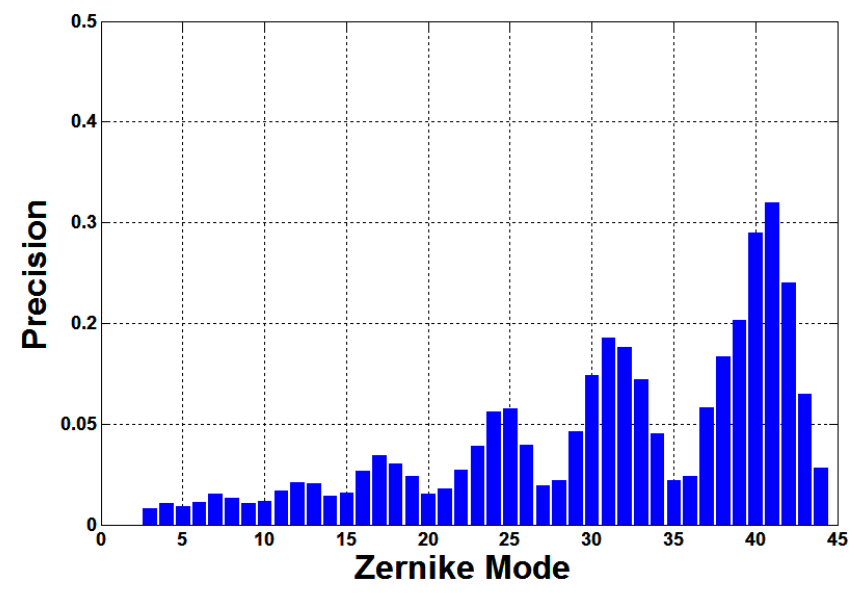

Fig. 5. The correction capability of wavefront errors of the 169-element PZT DM. 


\section{J. Zhao et al.}

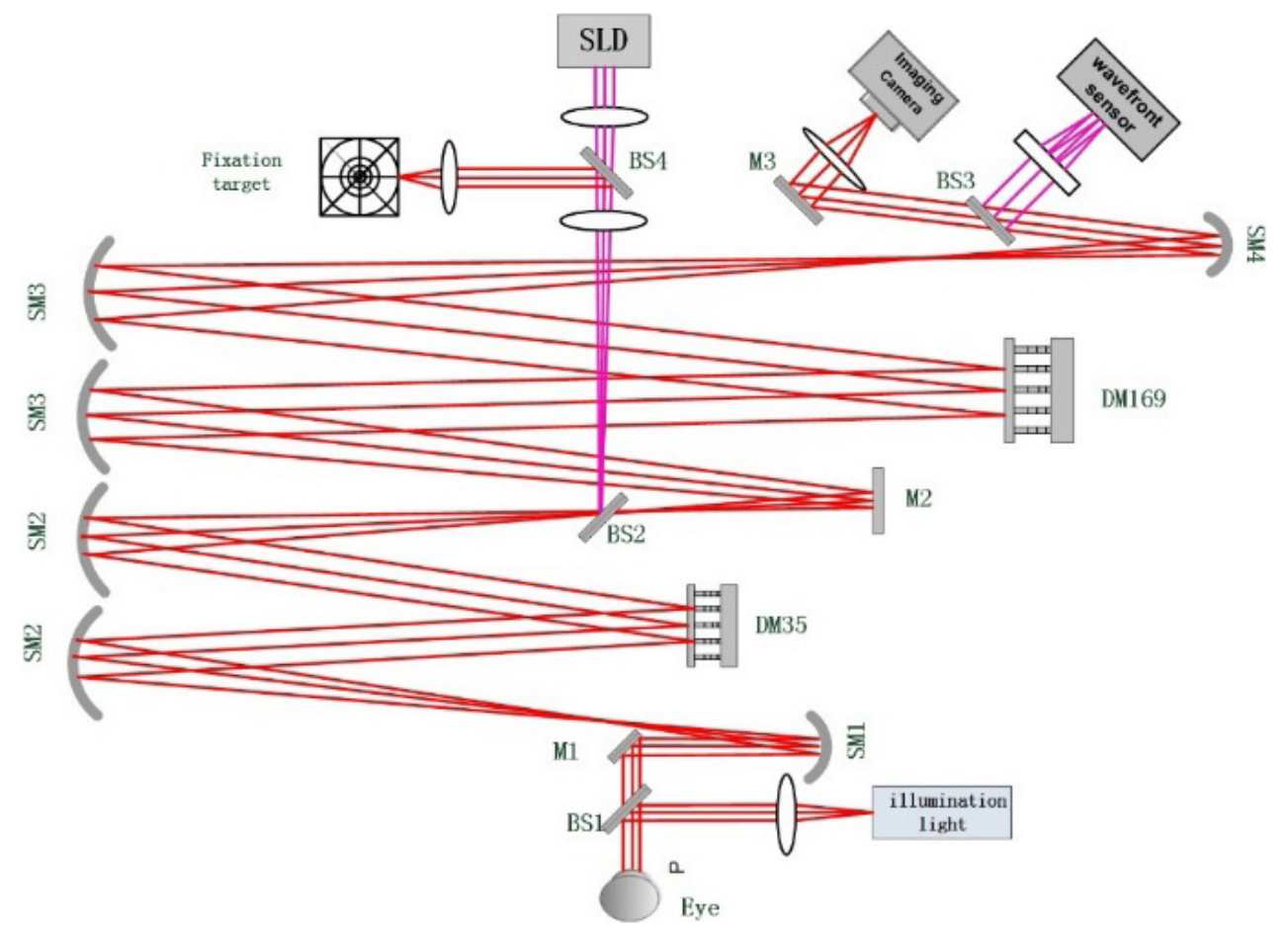

Fig. 6. Schematic diagram of dual DM flood-illumination system for retinal imaging. SLD super-luminescent diode; SM spherical mirror: SM1 $(f=180 \mathrm{~mm})$, SM2 $(f=600 \mathrm{~mm})$, SM3 $(f=1050 \mathrm{~mm})$, SM4 $(f=195 \mathrm{~mm})$; BS beam splitter; M mirror; DM deformable mirror.

region selection, (3) fiber-based light source and scientific-grade CCD for retinal imaging. The AO sub-system was the main part of the high-resolution retinal imaging system. This sub-system consisted of a beacon light source, a Shack-Hartmann wavefront sensor (SHWS) for wavefront sensing and two DMs mentioned above for wavefront compensation. To reduce the speckle effects, a super luminescent diode (SLD; $\lambda=798 \mathrm{~nm}, \Delta \lambda=18.8 \mathrm{~nm}$, $P=10 \mathrm{~mW}$ ) was used as beacon light source in the AO system. It was placed after the bimorph DM so that the incoming light can be pre-compensated. The wavefront error was measured with a SHWS consisting of a $16 \times 16$ lenslet array $(f=15 \mathrm{~mm}$; lenslet diameter $=0.4 \mathrm{~mm}$ ) which sampled the exiting wavefront across a 6 -mm pupil and a CCD camera at its focal plane.

The bimorph DM, PZT DM and SHWS were matched carefully as shown in Fig. 7. In order to obtain optimal aberrations correction performance and AO closed-loop stability with the $16 \times 16$ SHWS used for 35 -element bimorph $\mathrm{DM},{ }^{37}$ the layout analysis was conducted. According to the results of layout analysis, the corner actuators was discarded and only the rest 145 actuators were actually used

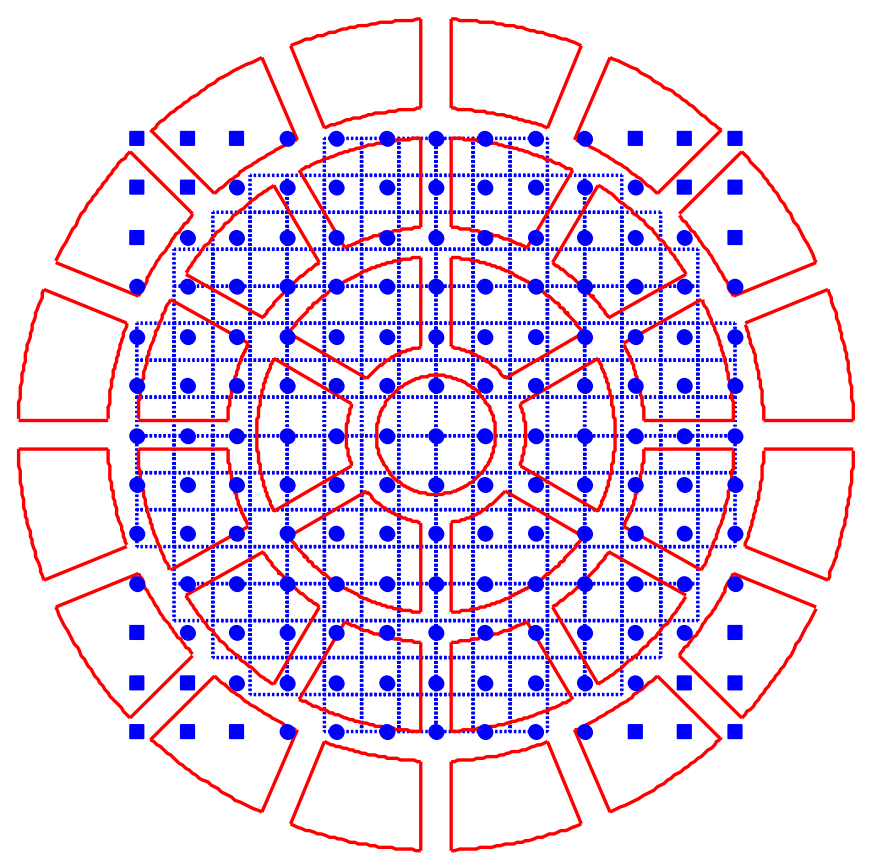

Fig. 7. Layout of bimorph DM, PZT DM and SHWS. The dotted squares represent the lenslets used to sample the pupil, the fan-shaped lines are the locations of the bimorph DM actuators, and the circles show the PZT DM actuator positions and the little squares in the four corners means the PZT DM actuators which are not used in the system. 
for the layout. The two DMs were conjugated to the pupil of the eye. A set of spherical mirrors was used as the relay optics to match in size the $35-\mathrm{mm}$ PZT $\mathrm{DM}$ with the 20-mm bimorph DM and a 6-mm pupil. The 35-element bimorph DM was used to correct large amplitude, LOAs and the residual HOAs were corrected by the 169-element PZT DM. A Zernike mode decomposition decoupling control algorithm was used for dual DMs AO system. ${ }^{38}$

\subsection{Retinal imaging}

The AO system was first tested by correcting the aberrations of the system itself and trial lenses. A flat mirror was placed at the position of the eye. The system was closed-loop with the beacon light and the light reflected by the mirror was focused at the imaging camera. To test the correction accuracy and correction range of the system, trail lenses with different power were inserted into the system and then corrected by DMs. The system could correct large aberrations up to \pm 8.0 diopters of defocus and \pm 4.5 diopters of cylindrical aberrations, while the residual wavefront error was about $0.04 \mu \mathrm{m}$.

We performed retinal imaging in 15 randomly recruited human subjects including eyes in normal group and eyes in abnormal group according to the correction range of the system. The subjects aged between 18 and 44 years and they had refractive errors between 0 and 8.0 diopters. Informed consent was obtained after a full explanation of the procedures and consequences of this study. Pupil was dilated with $1 \%$ cyclopentolate solution before data collection. Typical imaging results were shown as follows.

In order to evaluate the performance of the dual DM system for eyes in normal group, retinal images of Subject LB ( -2.75 diopter defocus, -0.5 diopter astigmatism, age 26) with healthy eyes were obtained. Figure 8 shows the RMS wavefront errors and corresponding SR over iterations. Figure 9 shows the retinal images acquired at the same retinal position with three different modes and the normalized power spectra of images. As shown in Figs. 9(a)-9(c), retinal cone mosaic was clearly resolvable only with dual DMs correction, which was consistent with the analysis of the requirement for $\mathrm{AO}$ correction in normal group.

As to evaluate the performance of the dual DM system for eyes in abnormal group, retinal images of Subject LL ( -2.75 diopter defocus, -0.5 diopter

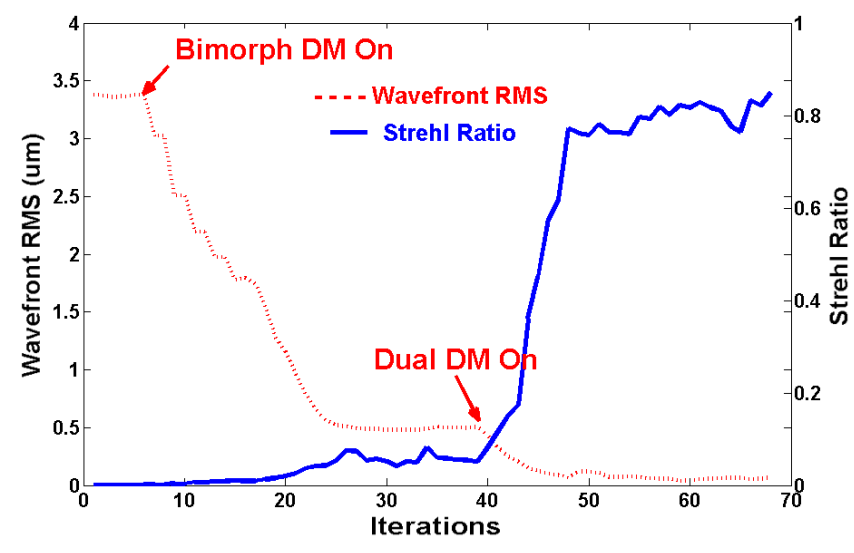

Fig. 8. The RMS wavefront errors and corresponding SR over iterations for Subject LB.

astigmatism, age 18) were obtained. All the subjects were recruited in our laboratory, they had no ocular pathologic features other than refractive error. However, the distribution of HOAs in Subject LL was similar to the statistics of HOAs in abnormal group. Figure 10(a) shows the HOAs of Subject LL. The HOAs RMS is $0.68 \mu \mathrm{m}$, which was approximate

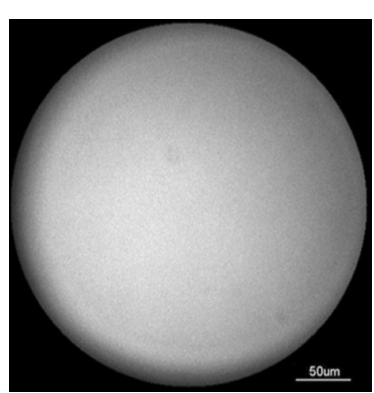

(a)

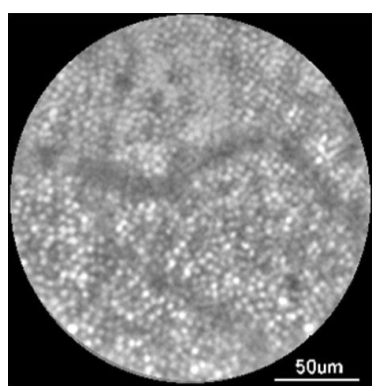

(c)

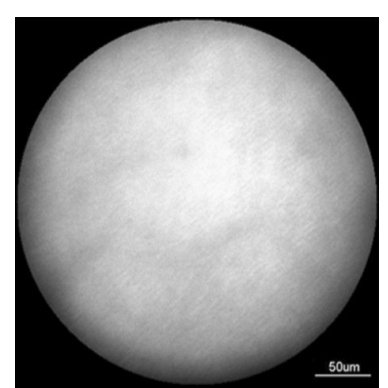

(b)

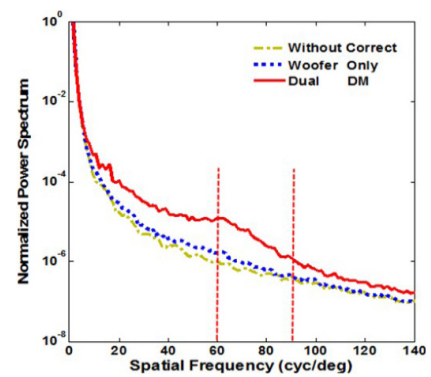

(d)
Fig. 9. Retinal imaging results of Subject LB and the normalized power spectra of images. (a)-(c) are the retinal images obtained without AO correction, with Bimorph DM corrected and with dual DMs corrected, respectively, the imaging area is at the retinal location of $2^{\circ} \mathrm{Nasal}$ and $2^{\circ}$ Superior and the field of view is $1^{\circ}$. (d) was the normalized power spectra of images (a) $-(\mathrm{c})$. 


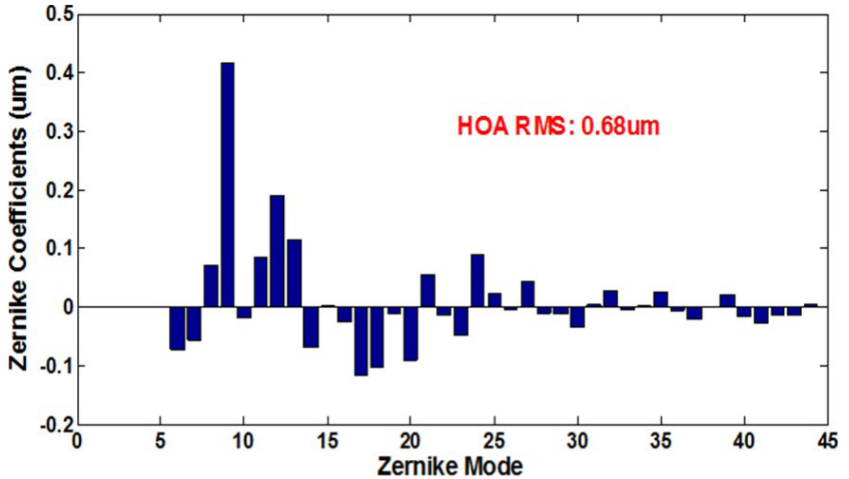

(a)

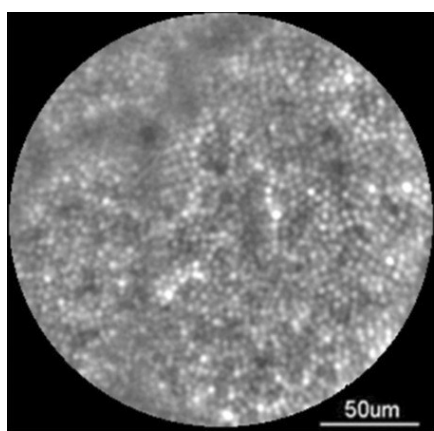

(b)

Fig. 10. HOAs of Subject LL (a) and the retinal image with dual DMs correction (b).

to value of $0.7 \mu \mathrm{m}$ in abnormal group. As shown in Fig. 10(b), high-resolution retinal image of Subject LL could be obtained as well, which proved that the dual-DM system could meet the requirement for $\mathrm{AO}$ correction in the abnormal group.

In conclusion, the retinal imaging results showed that the dual DM system could meet the requirement for $\mathrm{AO}$ correction in both normal and abnormal groups, which was consistent with the analysis of the requirement for $\mathrm{AO}$ correction of the Chinese eye's monochromatic aberrations.

\section{Conclusions}

In this paper, we collected the monochromatic wave aberration of 332 healthy eyes and 344 diseased eyes in Chinese population across a $6.0-\mathrm{mm}$ pupil. The aberration statistics of Chinese eyes including healthy eyes and diseased eyes were analyzed, and some differences of aberrations between the Chinese and European race were concluded. On this basis, the requirement for $\mathrm{AO}$ correction of the Chinese eye's monochromatic aberrations was analyzed. The result showed that a stroke of $20 \mu \mathrm{m}$ and ability to correct aberrations up to the 8th Zernike order were needed for reflective wavefront correctors to achieve near diffraction-limited imaging in both groups for a reference wavelength of $550 \mathrm{~nm}$ and a pupil diameter of $6.0 \mathrm{~mm}$. Then an AO flood-illumination system was established, and high-resolution retinal imaging in vivo was achieved for Chinese eye in both groups, which confirmed the analysis that we done above. This work could be used to guide AO ophthalmoscope design for large Chinese population application.

\section{Acknowledgments}

This study has been supported by the National Key Scientific Instrument and Equipment Development Project (Grant Nos. 2012YQ120080 and 2013YQ49085903), the National Natural Science Foundation of China (Grant No. 61378064) and the National High Technology Research and Development Program of China (Grant No. 2015AA020510). The authors would like to thank the colleagues in West China Hospital for helping to collect the raw data. We would like to thank Professor Wenhan Jiang for his critical comments and thoughtful suggestions. We also thank all subjects for their positive support in this work.

\section{References}

1. J. Liang, D. R. Williams, D. T. Miller, "Super healthy vision and high-resolution retinal imaging through adaptive optics," J. Opt. Soc. Am. A $\mathbf{1 4}$ (11), 2884-2892 (1997).

2. D. R. Williams, "Imaging single cells in the living retina," Vis. Res. 51, 1379-1396 (2011).

3. A. Roorda, F. Romero-Borja, W. J. Donnelly III, H. Queener, T. J. Hebert, M. C. W. Campbell, "Adaptive optics scanning laser ophthalmoscopy," Opt. Express 10(9), 405-412 (2002).

4. B. Hermann, E. J. Fernandez, A. Unterhuber, H. Sattmann, A. F. Fercher, W. Drexler et al., "Adaptive-optics ultrahigh-resolution optical coherence tomography," Opt. Lett. 29(18), 2142-2144 (2004).

5. Y. Zhang, J. Rha, R. S. Jonnal, D. T. Miller, "Adaptive optics parallel spectral domain optical coherence tomography for imaging the living retina," Opt. Express 13(12), 4792-4811 (2005). 
6. D. X. Hammer, R. D. Ferguson, C. E. Bigelow, N. V. Iftimia, T. E. Ustun, S. A. Burns, "Adaptive optics scanning laser ophthalmoscope for stabilized retinal imaging," Opt. Express 14(8), 3354-3367 (2006).

7. D. C. Gray, W. Merigan, J. I. Wolfing, B. P. Gee, J. Porter, A. Dubra, T. H. Twietmeyer, K. Ahmad, "In vivo fluorescence imaging of primate retinal ganglion cells and retinal pigment epithelial cells," Opt. Express 14(16), 7144-7158 (2006).

8. D. Merino, C. Dainty, "Adaptive optics enhanced simultaneous en-face optical coherence tomography and scanning laser ophthalmoscopy," Opt. Express 14(8), 3345-3353 (2006).

9. Y. Zhang, S. Poonja, A. Roorda, "MEMS-based adaptive optics scanning laser ophthalmoscopy," Opt. Lett. 31(9), 1268-1270 (2006).

10. S. A. Burns, R. Tumbar, A. E. Elsner, D. Ferguson, D. X. Hammer, "Large-field-of-view, modular, stabilized, adaptive-optics-based scanning laser ophthalmoscope," J. Opt. Soc. Am. A 24(5), 13131326 (2007).

11. J. E. Kim, M. Chung, "Adaptive optics for retinal imaging: Current status," Retina 33(8), 1483-1486 (2013).

12. D. Scoles, Y. N. Sulai, A. Dubra, "In vivo dark-field imaging of the retinal pigment epithelium cell mosaic," Biomed. Opt. Express 4(9), 1710-1723 (2013).

13. E. A. Rossi, P. Rangel-Fonseca, K. Parkins, W. Fischer, L. R. Latchney, M. A. Folwell, D. R. Williams, A. Dubra, M. M. Chung, "In vivo imaging of retinal pigment epithelium cells in age related macular degeneration," Biomed. Opt. Express 4(11), 2527-2539 (2013).

14. J. Zhang, Q. Yang, K. Saito, K. Nozato, A. Roorda, D. R. Williams, E. A. Rossi, "An adaptive optics imaging system designed for clinical use," Biomed. Opt. Express 6(8), 2120-2137 (2015).

15. X. Zhou, P. Bedggood, B. Bui, C. T. O. Nguyen, Z. He, A. Metha, "Contrast-based sensorless adaptive optics for retinal imaging," Biomed. Opt. Express 6 (9), 3577-3595 (2015).

16. E. J. Fernandez, I. Iglesias, P. Artal, "Closed-loop adaptive optics in the human eye," Opt. Lett. 26(10), 746-748 (2001).

17. D. T. Miller, A. Roorda, Adaptive optics in retinal microscopy and vision, Handbook of Optics, 3rd Edition, p. 553, McGraw Hill, New York (2010), pp. $553-554$.

18. R. Zawadzki, S. Choi, "Adaptive optics-optical coherence tomography: Optimizing visualization of microscopic retinal structures in three dimensions," J. Opt. Soc. Am. A 24(5), 1373-1383 (2007).

19. D. C. Chen, S. M. Jones, D. A. Silva, S. S. Olivier, "High-resolution adaptive optics scanning laser ophthalmoscope with dual deformable mirrors," $J$. Opt. Am. A 24(5), 1305-1312 (2007).

20. W. Zou, S. Burns, "Testing of Lagrange multiplier damped least-squares control algorithm for woofertweeter adaptive optics," Appl. Opt. 51(9), 11981208 (2012).

21. W. Zou, X. Qi, S. Burns, "Woofer-tweeter adaptive optics laser ophthalmoscopic imaging based on Lagrange-multiplier damped least-squares algorithm," Biomed. Opt. Express 2(7), 1986-2004 (2011).

22. D. X. Hammer, R. D. Ferguson, M. Mujat, A. Patel, E. Plumb, N. Iftimia, T. Y. P. Chui, J. D. Akula, A. B. Fulton, "Multimodal adaptive optics retinal imager: Design and performance," J. Opt. Soc. Am. A 29(12), 2598-2607 (2012).

23. J. Jacob, M. Paques, V. Krivosic, B. Dupas, A. Couturuer, C. Kulcsar, R. Tadayoni, P. Massin, A. Gaudric, "Meaning of visualizing retinal cone mosaic on adaptive optics images," Am. J. Ophthalmol. 159(1), 118-123 (2015).

24. M. Mujat, A. Patel, N. Iftimia, R. D. Ferguson, Compact adaptive optics line scanning retinal imager: Closer to the clinic, SPIE Photonics, San Francisco, CA, 1-6 February 2014.

25. J. Porter, A. Guirao, I. G. Cox, D. R. Williams, "Monochromatic aberrations of the human eye in a large population," J. Opt. Soc. Am. A 8(8), 17931803 (2001).

26. L. N. Thibos, A. Bradley, X. Hong, "A statistical model of the aberration structure of healthy, well corrected eyes," Ophthal. Physiol. Opt. 22(5), 427433 (2002).

27. L. N. Thibos, X. Hong, A. Bradley, X. Cheng, "Statistical variation of aberration structure and image quality in a healthy population of healthy eyes," J. Opt. Soc. Am. A 19(12), 2329-2348 (2002).

28. L. Wang, D. D. Koch., "Ocular higher-order aberrations in individuals screened for refractive surgery," J. Cataract Refract. Surg. 29(10), 18961903 (2003).

29. T. O. Salmon, C. van de Pol, "Healthy-eye Zernike coefficients and root-mean-square wavefront errors," J. Cataract Refract. Surg. 32(12), 2064-2074 (2006).

30. N. Maeda, T. Fujikado, T. Kuroda, T. Mihashi, Y. Hirohara, K. Nishida, H. Watanabe, Y. Tano, "Wavefront aberrations measured with HartmannShack sensor in patients with keratoconus," Ophthalmology 109(11), 1996-2003 (2002).

31. S. Pantanelli, S. MacRae, T. M. Jeong, G. Yoon, "Characterizing the wave aberration in eyes with keratoconus or penetrating keratoplasty using a high-dynamic range wavefront sensor," Ophthalmology 114(11), 2013-2021 (2007). 
32. A. Hartwig, D. A. Atchison, "Analysis of higher order aberrations in a large clinical population," Invest. Ophthalmol. Vis. Sci. 53(12), 7862-7870 (2012).

33. E. K. G. Au, T. H. Tay, M. K. Lim, "Race, culture and myopia in 110,236 young Singaporean males," Singapore Med. J. 34(1), 29-32 (1993).

34. F. Thorn, R. Held, L. L. Fang, "Orthogonal astigmatic axes in Chinese and Caucasian infants," Invest. Ophthalmol. Vis. Sci. 28(1), 191-194 (1987).

35. A. Carkeet, H. D. Luo, L. Tong, S. M. Saw, D. T. H. Tan, "Refractive error and monochromatic aberrations in Singaporean children," Vis. Res. 42(14), 1809-1824 (2002).
36. L. N. Thibos, R. A. Applegate, J. T. Schwiegerling, R. Webb, VSIA Standards Taskforce Members, "Standards for reporting the optical aberrations of eyes," J. Refract. Surg. 18(5), 652-660 (2002).

37. Y. Dai, L. Zhao, F. Xiao, H. Zhao, H. Bao, H. Zhou, Y. Zhou, Y. Zhang, "Adaptive optics vision simulation and perceptual learning system based on a 35-element bimorph deformable mirror," Appl. Opt. 54(5), 979-985 (2015).

38. W. Liu, L. Dong, P. Yang, X. Lei, H. Yan, B. Xu, "A Zernike mode decomposition decoupling control algorithm for dual deformable mirrors adaptive optics system," Opt. Express 21(20), 23885-23895 (2013). 